\title{
Heart failure: a special issue
}

\author{
Jolanda van der Velden • Pieter P. de Tombe
}

Received: 30 April 2014 / Accepted: 5 May 2014 / Published online: 14 May 2014

(C) Springer-Verlag Berlin Heidelberg 2014

Heart failure is a clinical syndrome in which the heart is unable to deliver the cardiac output that is required to meet the body's demand at the prevailing hemodynamic status [1]. The etiology of heart failure is diverse, caused by factors either external to the heart (increased mechanical stress due to, for example, hypertension or valve pathology) or by internal factors (for example coronary artery disease). Of interest, a significant proportion of patients present with an idiopathic cardiomyopathy; it is now recognized that many, if not all, of these cases may be due to inherited mutations, mainly in genes coding for sarcomeric proteins. A subset of patients may also develop heart failure secondary to cardiac infections. The heart failure syndrome can be further characterized in two classes of patients with a roughly equal prevalence: those that present with reduced cardiac contractile function (heart failure with reduced ejection fraction or systolic heart failure) and a second group of patients in whom cardiac contractile function is preserved, but, instead, cardiac filling is impaired (heart failure with preserved ejection fraction, or diastolic heart failure); the identification of the latter group of patients as a distinctly separate group is a relatively recent notion.

The overall mortality rate for acute heart failure, notably as caused by events such as acute myocardial infarction, has been steadily declining over the past years. However, despite modern and optimal treatment, the prognosis for heart failure has not improved significantly over the past decades. In fact,

J. van der Velden

Laboratory for Physiology, Institute for Cardiovascular Research, VU University Medical Center, Van der Boechorststraat 7 , 1081BT Amsterdam, The Netherlands

e-mail: j.vandervelden@vumc.nl

P. P. de Tombe $(\square)$

Department of Cell and Molecular Physiology, Stritch School of

Medicine, Loyola University Chicago, 2160 South First Ave.,

Maywood, IL 60153-5500, USA

e-mail: pdetombe@luc.edu the life expectancy for patients suffering from end-stage heart failure rival, and in some cases even exceeds, that of many malignancies. Moreover, treatment for heart failure with preserved ejection fraction (diastolic heart failure) based on strategies that were developed for systolic heart failure has proven to be ineffective. Thus, given the rise in the prevalence and incidence of heart failure, the dismal prognosis, and the tremendous financial burden generated, it is clear that new therapeutic strategies are desperately needed to combat the heart failure syndrome.

Accordingly, we have focused this special issue of Pflügers Archiv, European Journal of Physiology on heart failure to provide an update on the current status of knowledge in the field. We have invited world leaders and experts on the many aspects that play important roles in this syndrome, such as genetic mutations, transcription regulation, mechanosignaling, cellular signal transduction, extracellular matrix signaling, and excitation-contraction coupling. In addition, reviews are included that cover topics such as proteomic analysis as well as the current status of new treatment avenues based on, for example, gene therapies or novel small organic compounds.

As evidenced by the contributions in this special issue, significant progress has recently been made, both in identifying basic mechanisms underlying the heart failure syndrome, and in potential ways to either intervene or reverse cellular dysregulation in heart failure. With this expanding "toolbox" of basic understanding, it is expected that these novel findings can soon be translated to the bedside so as to deliver the new therapeutic strategies that are so desperately needed by the millions of patients suffering from heart failure.

\section{Reference}

1. Heart disease and stroke statistics - 2014 update. A report from the American Heart Association. Circulation. 2014; 129: e28-e292 